\title{
Investigation of the Causal Association between Long-Chain n-6 Polyunsaturated Fatty Acid Synthesis and the Risk of Type 2 Diabetes: A Mendelian Randomization Analysis
}

\author{
Michael A. Zulyniak ${ }^{a}$ Harriett Fuller $^{\mathrm{a}}$ Mark M. Iles $^{\mathrm{b}, \mathrm{c}}$ \\ a School of Food Science and Nutrition, Nutritional Epidemiology Group, University of Leeds, Leeds, UK; \\ b Leeds Institute of Medical Research, University of Leeds, Leeds, UK; ' Leeds Institute for Data Analytics, \\ University of Leeds, Leeds, UK
}

\section{Keywords \\ Mendelian randomization - Polyunsaturated fatty acid . Omega · FADS · Glycemia · MAGIC · UK Biobank · CHARGE · GIANT}

\begin{abstract}
Background: Globally, 1 in 11 adults has diabetes mellitus, and most of these cases are type 2 diabetes (T2D). The risk of T2D is influenced by many factors, including diet. The synthesis of long-chain n-6 polyunsaturated fatty acids (LC n-6 PUFA) has been posited as a risk factor for T2D; however, its causal role is uncertain. Aim: To test the causal effect of LC n-6 PUFA synthesis on insulin resistance and transgenerational T2D risk in a large cohort of men and women. Methods: Two-sample mendelian randomization (MR) was conducted to evaluate the effect of low or high levels of LC n-6 PUFA synthesis on glycemia and development of T2D in the UK Biobank ( $n=463,010)$ and Meta-Analysis of Glucose- and Insulin-Related Traits Consortium (MAGIC; $n=5,130$ ) cohorts. The increased likelihood of a predisposition to low or high LC n-6 PUFA synthesis and the risk of T2D was also investigated using the participants' siblings and parents. In MR-Base, 4 genetic variants associated with LC n-6 PUFA syn-
\end{abstract}

thesis were found ( $p<10^{-8}$ ). After pruning, 1 variant (rs174547) on the FADS1 gene was retained. Results: Lower LC n-6 PUFA synthesis and abundance (per \% unit decrease) are associated with small reductions in the insulin disposition index $\left(-0.038 \pm 0.012 \mathrm{mM}^{-1} ; p=0.002\right)$ within MAGIC. In the UK Biobank, we report negligible effects of low n-6 PUFA synthesis on the odds of T2D (OR $<1 \%$; $p<0.05)$. Additionally, reduced LC n-6 PUFA synthesis does not appear to be a contributor to familial T2D risk. No significant association was observed between LC n-6 PUFA synthesis and BMI. Conclusion: In a primarily white European population, LC n-6 PUFA synthesis is not a major contributor to T2D risk.

(c) 2020 The Author(s)
Published by S. Karger AG, Basel

\section{Introduction}

Globally, nearly 1 in 11 adults are diabetic, and 90\% of them have type 2 diabetes (T2D). T2D is characterized by a deterioration of $\beta$-cell function, often preceded by insulin resistance [1]. A number of mechanisms underlying $\beta$-cell dysfunction have been proposed, including genetics, bodyweight and adiposity, smoking, inflammation, physical activity, and diet [2-5]. 


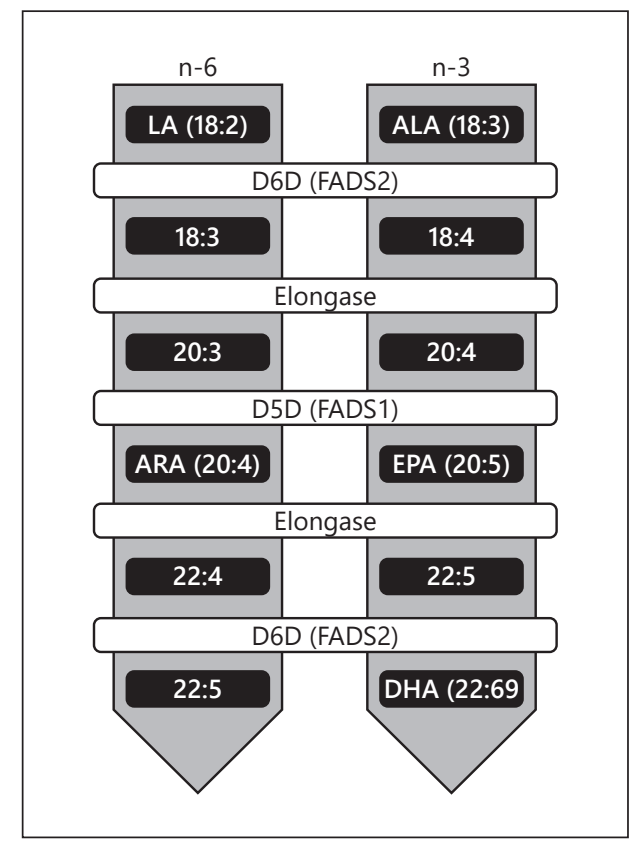

Fig. 1. n-6 and n-3 PUFA synthesis. LC n-6 and n-3 PUFA can be synthesized from precursor PUFA (LA or ALA) by alternating reactions of desaturation and elongation. Desaturation is driven by $\delta-6$ and $\delta-5$ desaturase (D5D and D6D), coded by fatty acid desaturase (FADS1 or FADS2), while elongation is driven by elongase, coded by elongation 2 or 5 (ELOVL 2 or ELOVL 5).

Dietary fats have been positioned as an important risk factor for T2D, with polyunsaturated fatty acids (PUFA) demonstrating a strong association with glucose metabolism [6]. A meta-analysis of 102 clinical trials $(n=4,220$ adults) reported that isocaloric replacement of carbohydrates with PUFA improved glycemia levels $(-0.11$ $\mathrm{HbA} 1 \mathrm{c} \%$; $95 \% \mathrm{CI}-0.17$ to $-0.05 ;-1.6 \mathrm{pmol} / \mathrm{L}$ fasting insulin; $95 \%$ CI -2.8 to -0.4$)$ and insulin secretion $(+0.5$ $\mathrm{pmol} / \mathrm{L} / \mathrm{min}$; $95 \%$ CI 0.2 to 0.8 ) more significantly than replacement with saturated (SFA) or monounsaturated (MUFA) [6] Observational studies have reported similar results and estimated that a low dietary PUFA consumption may be responsible for up to $20 \%$ (population-attributable fraction) of T2D cases [7]. However, heterogeneity between studies, insufficient power, and confounding [8$10]$ limit our interpretation of these results.

Of particular interest is the rate of synthesis and abundance of long-chain n-6 PUFA (LC n-6 PUFA), i.e., arachidonic acid (ARA; 20:4 n-6), from shorter precursor n-6 PUFA such as linoleic acid (LA; 18:2 n-6) (Fig. 1). Although the specific mechanisms remain elusive, it has been observed that exposure of primary $\beta$-cell cultures to elevated levels of ARA by (1) exogenous application or (2)

The Causal Association between LC n-6

PUFA and T2D: An MR Study impeding the conversion of ARA to eicosanoids stimulates insulin secretion from human islets $[11,12]$, suggesting that the metabolism and abundance of LC n-6 PUFA has a direct effect on insulin secretion. Moreover, a nested case-control study ( $n=2,787$, with 673 cases of T2D) within the European Prospective Investigation into Cancer and Nutrition (EPIC)-Potsdam cohort reported that higher rates of ARA synthesis resulted in a $54 \%$ reduction in the risk of T2D ( $\mathrm{RR}=0.46$; $95 \%$ CI $0.31-0.70$; $p$-trend $<0.0001$ ), compared to the lowest quintile, in their fully adjusted model [13]. Collectively, this suggests that LC n-6 PUFA are associated with the risk of T2D and may be driven by ARA acting on $\beta$-cell insulin secretion; however, such studies are limited by confounding. Confounding is the uncertainty introduced by inaccurate or unaccounted variables (e.g., cooking method) [14, 15] that are linked to both the exposure (i.e., ARA) and the outcome (i.e., $\beta$-cell insulin secretion) [16].

To overcome the effects of confounding and provide evidence of a causal association, EPIC elected to use a mendelian randomization (MR) approach. Briefly, MR uses genetic variants associated with exposure as surrogate measures of that exposure (i.e., instrumental variables). The use of genetic variants (e.g., single-nucleotide polymorphisms; SNP) permits the inference of causality between these exposures and the outcome because genetic variants are (1) randomly assorted at meiotic segregation and (2) assigned at conception. In a way, this model resembles a life-long randomized controlled trial where the exposures and confounders (e.g., fish intake, fish oil supplementation, physical activity, and household income) are also randomly distributed $[16,17]$. The aim was for the MR analysis undertaken by the EPIC study to demonstrate a causally protective effect of ARA on T2D [13]. However, the wide confidence intervals $(R R=0.98$; 95\% CI 0.70-1.37) they attained suggested that, if an association exists, a larger sample would be needed to reflect the true causal association between n-6 PUFA synthesis and T2D. Two-sample MR [18] harnesses summary association results from nonoverlapping groups of individuals to estimate novel causal effects. This method increases the utility of large cohorts for MR studies because researchers are not reliant on a single cohort to provide exposure and outcome data; in 2-sample MR any 2 independent cohorts may be used - one to summarize the genetic variant-exposure association and another to summarize the genetic variant-outcome association. With this methodology and access to large cohorts with suitable genetic or exposure data, this approach can greatly increase statistical power. 
Table 1. Association between the SNP and the instrumental variable (rs174547) and n-6 polyunsaturated fatty acids (PUFAs)

\begin{tabular}{lllll}
\hline n-6 PUFA & $\beta$ & SE & $p$ value & $\mathrm{R}^{2}(\%)$ \\
\hline Linoleic acid (LA) & 1.47 & 0.05 & $5.00 \mathrm{E}-274$ & $7.6-18.1$ \\
$\gamma$-Linoleic acid (GLA) & -0.016 & 0.001 & $2.30 \mathrm{E}-72$ & $2.2-4.6$ \\
Di-homo- $\gamma$-linoleic acid (DGLA) & 0.36 & 0.01 & $2.60 \mathrm{E}-151$ & $8.7-11.1$ \\
Arachidonic acid (ARA) & -1.69 & 0.02 & $3 \mathrm{E}-971$ & $3.7-37.6$ \\
\hline
\end{tabular}

The SNP rs174547 (C/T) has a signifciant effect on n-6 PUFA levels, with an effect allele (C) frequency of 0.33 .

To build on previous evidence, we propose the use of 2-sample MR to assess the causal association between LC n-6 PUFA synthesis and the risk of T2D.

\section{Methods}

\section{Instrumental Variables}

We performed 2-sample MR using MR-Base [19] with summary data from publicly available GWAS databases (online suppl. Fig. 1; see www.karger.com/doi/10.1159/000509663 for all online suppl. material).

\section{Sample 1}

With MR-Base [19], instrumental variables (SNP) associated $\left(p<5 \times 10^{-8}\right)$ with plasma levels (\% total fatty acids) of ARA were identified. From the Cohorts for Heart and Aging Research in Genomic Epidemiology Consortium (CHARGE; $n=8,631$ white Europeans; 55\% women) [20] - comprising the Atherosclerosis Risk in Communities (ARIC), Coronary Artery Risk Development in Young Adults (CARDIA), Cardiovascular Health Study (CHS), Invecchiare in Chianti (InCHIANTI), and Multi-Ethnic Study of Atherosclerosis (MESA) - the following 4 SNP were identified: rs174547, rs102275, rs1741, and rs16829840. From a nested casecontrol study of myocardial infarction within the Singapore Chinese Health Study (SCHS; $n=1,540$ ) the following 2 SNP were identified: rs174577 and rs174548. Of the 6 SNP identified, the following 2 were removed because their biological function and mechanism of association with ARA were unknown, which risks violation of MR (i.e., no horizontal pleiotropy between SNP and outcome): rs1741, a non-coding intron variant strongly associated with vitamin $\mathrm{B}_{6}$-dependent decarboxylase (it is unknown whether this variant alters carboxylase expression); and rs16829840, a noncoding transcript variant of transmembrane protein (TMEM39A) with an uncertain function and cellular location [21]. Therefore, only 4 SNP (rs174547, rs102275, rs174577, and rs174548) progressed and were evaluated for inclusion in 2-sample MR.

\section{Sample 2}

Using previously published data within MR-Base, summary associations for each of the 4 SNP were obtained for: (1) insulin disposition index (DI) in the Meta-Analysis of Glucose- and InsulinRelated Traits Consortium (MAGIC; white European men and women; $n=5,130)$ and (2) self-reported diagnosis of T2D in the
UK Biobank (95\% white European men and women, $n=463,010$ ). The DI, calculated following an oral glucose tolerance test, is associated with beta-cell function $(p<0.001)$ and is a predictor of future T2D (Area Under the Curve $=0.86$; 95\% CI: $0.82,0.89$ ) [22], whereby a lower or reduction in a person's DI is associated with a shift from normal glucose tolerance to impaired glucose metabolism and an increased risk of T2D.

In case an SNP was not present in MAGIC or the UK Biobank, a proxy SNP in high linkage disequilibrium $\left(r^{2} \geq 0.80\right)$ would be identified; however, proxy SNP were not required as all SNP were in both databases. Palindromic SNP were permitted with a minor allele frequency threshold of 0.30 to allow for a high degree of confidence that sample 1 and sample 2 datasets were coded in the same direction [23]. SNP were tested for independence and clumped $\left(r^{2}<0.001\right)$. Using MR-Base and the ld_matrix function (TwoSampleMR) in R (v.3.5.1), we observed LD with $R^{2}>0.92$ between SNP within the 1000 Genomes Project of European samples. As depicted in Figure 1, the genes/enzymes for elongation and desaturation are shared by the $n-6$ and $n-3$ pathways; therefore, a single SNP may be associated with multiple PUFA. To negotiate this link, we used existing summary data [20] to confirm that our instrumental variable (rs174547) explained a very high degree of variation of $n-6$ PUFA levels and, comparatively, little variation of n-3 PUFA levels. While the effect of rs174547 is significant across numerous PUFA, the magnitude of the effect of rs174547 on n-6 PUFAs is significantly greater (up to 22 -fold more) [24], suggesting that rs 174547 is primarily a regulator of $\mathrm{n}-6$ PUFA synthesis. Underlying this association is the large pool of LA, a short chain n-6 PUFA, which constitutes $\approx 90 \%$ of all dietary PUFA and precursor of endogenously synthesized long-chain n-6 PUFA [25]. Furthermore, as shown in Table 1, we see that while rs174547 may not be an ideal instrumental variable for a single n-6 PUFA, that it is most strongly associated with n-6 PUFAs at opposite ends of the pathway and in opposite directions - i.e., higher levels of LA (an essential shortchain n-6 PUFA; $\beta=+1.47[0.05] \%$ total fatty acids, $p=5.0 \times$ $10^{-274}$ ) and lower levels of arachidonic acid (a major LC n-6 PUFA and precursor of eicosanoids; $\beta=-1.69[0.02] \%$ total fatty acids, $p=3.0 \times 10^{-971}$ ). Therefore, we used rs174547 as an instrumental variable of n-6 PUFA synthesis and report all MR associations per $1 \%$ unit decrease in arachidonic acid of total plasma fatty acids (i.e., lower n-6 PUFA synthesis). This genetic variant demonstrates no association with common confounders of diabetes, such as selfreported "smoking" or "alcohol intake" in the UK Biobank [26]. In summary, rs174547 meets the 3 assumptions of MR [27], i.e.: (1) correlated with the study exposure (n-6 PUFA synthesis); 
Table 2. Causal association estimates of the effect of reduced n-6 PUFA synthesis on dysglycemia and the risk of type 2 diabetes

\begin{tabular}{|c|c|c|c|c|c|}
\hline \multirow[t]{2}{*}{ Outcome } & \multicolumn{5}{|c|}{ Wald estimate } \\
\hline & $\beta$ & SE & $p$ value & $n$ & OR $(95 \% \mathrm{CI})$ \\
\hline Insulin Disposition Index $\left(\mathrm{mM}^{-1}\right)$ & -0.038 & 0.012 & 0.002 & 5,318 & \\
\hline Participant T2D & 0.0005 & 0.0003 & 0.049 & 461,578 & $1.001(1.000-1.001)$ \\
\hline Mother T2D & 0.0008 & 0.0004 & 0.042 & 423,892 & $1.001(1.000-1.002)$ \\
\hline Father T2D & 0.0008 & 0.0004 & 0.054 & 400,687 & $1.001(1.000-1.002)$ \\
\hline Sibling T2D & 0.0005 & 0.0004 & 0.238 & 362,826 & $1.000(1.000-1.001)$ \\
\hline Body mass index $\left(\mathrm{kg} / \mathrm{m}^{2}\right)$ & -0.0015 & 0.0020 & 0.449 & 322,154 & \\
\hline
\end{tabular}

There is a causal association of a $1 \%$ reduction of n-6 PUFA synthesis (i.e., a 1\% reduction of ARA abundance) on Participant Disposition Index $\left(\mathrm{mM}^{-1}\right)$ and the risk of participant, parent, or sibling of type 2 diabetes (T2D). Diagnoses of T2D were self-reported by the primary particpant. SE, standard error.

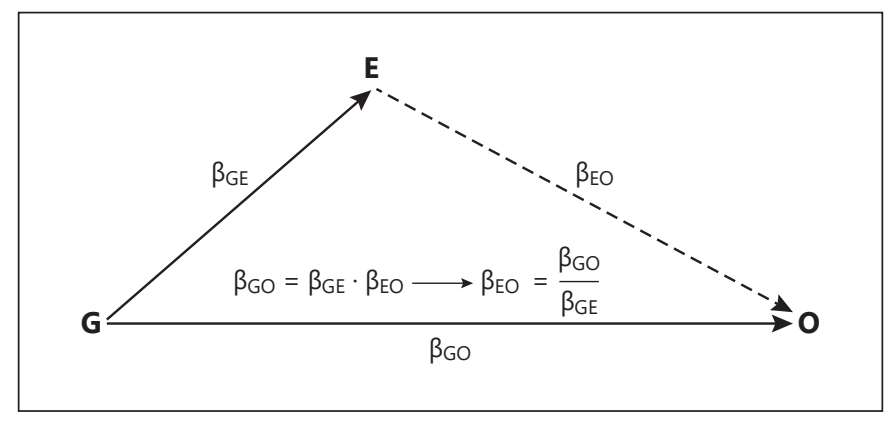

Fig. 2. Wald estimate. The vertices of the triangle represent: G, genetic variants (SNP) associated with our exposure and outcome; E, exposure of interest; and $\mathrm{O}$, outcome of interest. As such, the sides of the triangle represent: $\beta_{\mathrm{GE}}$, the association between specific SNP and the exposure; $\beta_{\mathrm{GO}}$, the association between specific SNP and the outcome; and $\beta_{\mathrm{EO}}$, the association between the exposure and the outcome. The Wald ratio states that if $\beta_{\mathrm{GE}}$ and $\beta_{\mathrm{EO}}$ are free of confounding, then their product is equal to $\beta_{\mathrm{GO}}$. Therefore, by rearranging this equation, $\beta_{\mathrm{EO}}$, the unconfounded association between n-6 PUFA and dysglycemia and T2D can be investigated.

(2) presents no association with other confounding factors (i.e., smoking and alcohol), and (3) offers no alternative independent biological pathway to modify the outcome (i.e., only via n-6 PUFA synthesis).

Within the UK Biobank, we also performed an exploratory analysis of family history of T2D. Briefly, given that at least one parent of any individual carrying the risk allele (T) of the rs174547 SNP must also carry the same risk allele, it can be expected that the effect of the SNP on n-6 PUFA synthesis and T2D risk must also be observed in at least one parent. Further, we expect siblings of a participant that carry the rs 174547 risk allele to also be more likely to carry this allele and present an association between n-6 PUFA and T2D risk. Data distinguishing between full and half siblings was not available.

Finally, to test whether an association between n-6 PUFA synthesis and T2D is mediated by changes in adiposity, we also investigated whether n-6 PUFA synthesis was causally associated with BMI in men and women within the GIANT consortium $(n=322,154)$.

The Causal Association between LC n-6

PUFA and T2D: An MR Study

\section{Statistical Methods}

Gathering our exposure (sample 1) and outcome (sample 2) summary results was the first step towards calculating 2-sample MR estimates of n-6 PUFA synthesis on dysglycemia or the odds of T2D. In the present study, the online tool mRnd (http://cnsgenomics.com/shiny/mRnd/) [28] estimated the study power to be over $90 \%$ and an $F$ statistic $>11$ to detect a significant difference $(p<0.05)$ in the risk of T2D of $5 \%$, assuming a conservative mean $r^{2}$ of 0.20 between our instrumental variable and exposure and a proportion of T2D in the UK Biobank of at least 5\% [29].

With a genetic instrument comprised of $1 \mathrm{SNP}$, the estimator for assessing a causal association between reduced n-6 PUFA synthesis and dysglycemia or T2D is the Wald estimate [30] (Fig. 2). This procedure relies on a relatively simple mathematical equation to calculate the causal effect of the exposure on the outcome. In line with the assumptions of $\mathrm{MR}$, the effect of $\beta_{\mathrm{GE}}$ (i.e., the difference in n-6 PUFA synthesis due to genetics/SNP) and $\beta_{\mathrm{EO}}$ (the effect of genetically-mediated n-6 PUFA synthesis on dysglycemia or T2D) are essentially free of confounding (due to random assortment of alleles at meiosis and conception) and horizontal pleiotropy. Therefore, $\beta_{\mathrm{GO}}$ (the direct effect of the SNP on dysglycemia or T2D) is simply the product of $\beta_{\mathrm{GE}}$ and $\beta_{\mathrm{EO}}\left(\beta_{\mathrm{GO}}=\beta_{\mathrm{GE}} \times \beta_{\mathrm{EO}}\right)$. Having already collected the estimates of $\beta_{\mathrm{GE}}$ and $\beta_{\mathrm{GO}}$ from the summary data above, the equation can be rearranged to solve for $\beta_{\mathrm{EO}}$, our primary research question, the causal association between n-6 PUFA synthesis and dysglycemia or T2D. Results were calculated per SD change in ARA but converted to percent unit change (i.e., 1\%) for easier interpretation.

\section{Results}

MR results are shown in Table 2. Firstly, in the MAGIC cohort, we report a small but significant association between a $1 \%$ reduction of n- 6 PUFA synthesis and the insulin DI $\left(-0.038 \mathrm{~mm}^{-1} ; p=0.002\right)$. Secondly, within the UK Biobank, we report very small but significant associations between a $1 \%$ reduction of n- 6 PUFA synthesis and the odds of $\mathrm{T} 2 \mathrm{D}$ in participants $(\mathrm{OR}=1.001 ; 95 \% \mathrm{CI}$ 
$1.000-1.001 ; p=0.049)$. Thirdly, in or exploratory analysis of familial risk of T2D, we report that mothers of individuals with reduced n-6 PUFA synthesis are only more likely to have had T2D by the smallest of margins (OR = 1.001 ; 95\% CI $1.000-1.002$; $p=0.042$ ), but not their fathers or siblings.

In our previous work, we observed reductions of n-6 PUFA synthesis of $\sim 1.7 \%$ following a 3-month supplementation of fish oils ( $2 \mathrm{~g}$ eicosapentaenoic acid and $1 \mathrm{~g}$ docosahexaenoic acid) in young and older men [31]. Therefore, we also present estimates of effect sizes which are proportional to a $1.7 \%$ reduction of n- 6 PUFA synthesis. For DI, this equates to a reduction of $-0.0665(0.021)$ $\mathrm{mM}^{-1}$. Put into context, where an increase of 1 unit of DI is associated with a $60 \%$ lower odds of developing T2D within 10 years $(\mathrm{OR}=0.40$; 95\% CI $0.25-0.66 ; p<0.001)$ [22], a reduction $-0.0665 \mathrm{mM}^{-1}$ is associated with a $6 \%$ increased risk of T2D within 10 years. For diagnosis of T2D, the difference in effect size between a $1 \%\left(\log \mathrm{OR}_{1 \%}=\right.$ 0.0005 ) and a $1.7 \%$ reduction in $n-6$ PUFA synthesis is negligible $\left(\log \mathrm{OR}_{1.7 \%}=0.00095\right)$. Put into perspective, within the UK Biobank where a high proportion of habitual fish oil intake was reported (31.2\%) [32], we estimate that fewer than $0.03 \%$ of cases of T2D may be attributed to fish oil supplementation (population attributable risk).

Finally, our MR model did not report a significant association between n-6 PUFA synthesis and BMI ( $p=$ 0.449 ) within the GIANT consortium.

\section{Discussion}

Using a 2-sample MR approach, we report that a reduction of n-6 PUFA synthesis is associated with T2D risk, but its effect is trivial. Additionally, we report that n-6 PUFA synthesis is not a major contributor to a family history of T2D.

With the relatively recent application of MR, the majority of analyses investigating the association between n-6 PUFA synthesis (or ARA abundance) and risk of T2D has been undertaken in large cohorts. Most recently, the Multi-Ethnic Study of Atherosclerosis (MESA) cohort ( $n$ $=6,282$ ) reported an inverse association between ARA levels and fasting insulin $(-0.272$ per SD; $p<0.001)$ and HOMA-IR $(-0.125$ per SD; $p=0.03)$ [33], which is in agreement with our findings and points toward a protective effect between ARA and T2D risk. However, our results disagree with a recent MR study that reported a small but positive causal association between ARA and T2D risk $\left(1.03\right.$; 95\% CI 1.02-1.05; $\left.p=2.51 \times 10^{-5}\right)$ with- in the DIAGRAM consortium [33]. As we both used comparable genetic summary statistics for estimating ARA levels, this may be an effect of population stratification, influenced by small differences in ethnic and geographic allele frequencies between the UK Biobank (multiethnic from the UK) and DIAGRAM (white Europeans from the UK, Finland, and the USA).

The primary aim of this study was to build on the results of a 1-sample MR study performed within the EPIC cohort 10 years ago [13], predating the introduction of 2-sample MR [18]. The EPIC population consisted of 2,653 men and women with genetic data related to SNP rs 174546 as an instrumental variable of n-6 PUFA synthesis (rather than rs 174547 as we have). The $r^{2}$ between rs 174546 and rs174547 is 1.0 [34], suggesting perfect linkage disequilibrium and that MR performed with either SNP should provide equivalent estimates. EPIC reported a risk ratio of 0.98 with wide confidence intervals (i.e., $0.70-1.37$ ), suggesting a low power despite the use of a strong instrumental variable (i.e., $F$ statistic $>10$ ) [16]. This is the strength of 2-sample MR, whereby a robust instrumental variable identified in one cohort is applied to a second (often larger) cohort with genetic-outcome data to improve power. We have taken this approach and report that our results fall within the confidence intervals of EPIC and now provide a more accurate representation of the negligible effect of n-6 PUFA synthesis on T2D risk.

Recent estimates from the DISCOTWIN consortium ( $n=34,166$ twin pairs) estimate the heritability of T2D to be $72 \%$ (95\% CI 61-78) [35]. Building on this, a GWAS of 69,142 Icelandic men and women demonstrated that, within a panel of 36 genetic variants associated with blood glucose, more than half were to some degree linked to familial heritability of fasting glucose. The FADS1 variant rs 174576, strongly correlated with $\mathrm{rs} 174547\left(r^{2}=0.94\right)$, was investigated and showed a small but not significant effect $(p=$ 0.069 ) on heritability of fasting glucose [36]. As an exploratory assessment, we investigated whether the parents and siblings of individuals who carry a genetic predisposition to lowered n-6 PUFA synthesis are also at an increased risk of T2D. Our analysis of parents of siblings suggests that rate of n-6 PUFA synthesis is not an important or impactful contributor to familial risk of T2D and familial risk is driven by other genetic and environmental factors.

The pathway by which n-6 PUFA affects glucose levels and diabetes risk is not well-characterized but it is commonly linked to high adiposity and circulating proinflammatory eicosanoids. Indeed, circulating levels of n-6 PUFA have been associated with an increased BMI across the lifespan $[37,38]$ and elevated levels of proinflamma- 
tory eicosanoids in unhealthy and healthy humans [39, 40]. Recently, two independent MR studies, one using the UK Biobank (13,982 T2D cases and 273,412 controls) and the other using both the Wellcome Trust Case Control Consortium and an Australian adult and adolescent cohort (22,669 T2D cases and 58,119 controls) confirmed a causative association between an elevated BMI and the risk of T2D, i.e., $\mathrm{OR}=1.37$ (95\% CI 1.12-1.68) per kilogram/square meter increase or OR $=1.47$ (95\% CI 1.17 $1.85)$ per $5-\mathrm{kg}$ increase $[41,42]$. Unfortunately, instrumental variables of proinflammatory eicosanoids have not been identified that are independent of PUFA levels so the causative association of proinflammatory eicosanoids on T2D is less well established. Therefore, we sought to investigate whether the effects of n-6 PUFA levels on T2D were driven by the effects of n-6 PUFA on adiposity. Our results were not significant and suggest that BMI and n-6 PUFA are associated with T2D through independent pathways.

The strength of MR is in its ability to minimize the effects of confounding; however, as with all studies, we cannot rule out the possible effects of unmeasured or latent confounders. Although our instrumental variable explained a very high proportion of variation of blood LA and ARA levels, we are unable to tease apart or isolate the effects of distinct PUFA using instrumental variables. However, by accepting this limitation and focusing on (1) the causative association of n-6 PUFA synthesis on T2D and (2) selecting genetic variants that are more influential towards n-6 (rather than n-3) PUFA synthesis we aimed to minimize this. We also tested our instrumental variable against the pleiotropic effect of BMI and found no association. Perhaps the greatest strength of the present study was the very large population, which allowed us to very precisely quantify the very small magnitude of effect of n-6 PUFA synthesis on the risk of T2D.

\section{Conclusion}

Evidence suggests that any heritable causal association between reduced LC n-6 PUFA synthesis and impaired glucose metabolism and progression towards T2D that does exist in a primarily white European population is negligible and likely of little importance in a clinical setting.

\section{Statement of Ethics}

This study assessed publicly available data from CHARGE, GIANT, MAGIC, SCHS, and the UK Biobank that is freely available on MR-Base. All of the studies and consortia accessed in the present study on MR-Base were approved by their respective ethics committee, and the subjects from all the cohorts provided written informed consent [43-47].

\section{Conflict of Interest Statement}

The authors have no conflict of interests to declare.

\section{Funding Sources}

M.A.Z. is currently funded by the Wellcome Trust.

\section{Author Contributions}

M.A.Z. conceived this study, analyzed the data, and drafted this paper. M.A.Z. and H.F. discussed and interpreted results. H.F. and M.I. provided critical feedback and revision of this work. All of the authors approved the final version of this work.

\section{References}

1 American Diabetes Association. Classification and diagnosis of diabetes: standards of medical care in diabetes: 2018. Diabetes Care. 2018 Jan;41 Suppl 1:S13-27.

2 Balk EM, Earley A, Raman G, Avendano EA, Pittas AG, Remington PL. Combined diet and physical activity promotion programs to prevent type 2 diabetes among persons at increased risk: a systematic review for the Community Preventive Services Task Force. Ann Intern Med. 2015 Sep;163(6):437-51.
3 Emdin CA, Khera AV, Natarajan P, Klarin D, Zekavat SM, Hsiao AJ, et al. Genetic Association of Waist-to-Hip Ratio With Cardiometabolic Traits, Type 2 Diabetes, and Coronary Heart Disease. JAMA. 2017 Feb; 317(6):626-34.

4 International Diabetes Federation. IDF diabetes atlas. 8th ed. Brussels: IDF; 2017.

5 Scott RA, Scott LJ, Mägi R, Marullo L, Gaulton KJ, Kaakinen M, et al.; DIAbetes Genetics Replication And Meta-analysis (DIAGRAM) Consortium. An expanded genome-wide association study of type 2 diabetes in Europeans. Diabetes. 2017 Nov;66(11): 2888-902.
6 Imamura F, Micha R, Wu JH, de Oliveira Otto MC, Otite FO, Abioye AI, et al. Effects of saturated fat, polyunsaturated fat, monounsaturated fat, and carbohydrate on glucose-insulin homeostasis: a systematic review and meta-analysis of randomised controlled feeding trials. PLoS Med. 2016 Jul; 13(7):e1002087.

7 Hu FB, Manson JE, Stampfer MJ, Colditz G, Liu S, Solomon CG, et al. Diet, lifestyle, and the risk of type 2 diabetes mellitus in women. N Engl J Med. 2001 Sep;345(11):790-7. 
8 Lankinen MA, Stančáková A, Uusitupa M, Ågren J, Pihlajamäki J, Kuusisto J, et al. Plasma fatty acids as predictors of glycaemia and type 2 diabetes. Diabetologia. 2015 Nov; 58(11):2533-44.

9 Yary T, Voutilainen S, Tuomainen TP, Ruusunen A, Nurmi T, Virtanen JK. Serum n-6 polyunsaturated fatty acids, $\Delta 5$ - and $\Delta 6$ desaturase activities, and risk of incident type 2 diabetes in men: the Kuopio Ischaemic Heart Disease Risk Factor Study. Am J Clin Nutr. 2016 May;103(5):1337-43.

$10 \mathrm{Wu} \mathrm{JH}$, Marklund $\mathrm{M}$, Imamura F, Tintle $\mathrm{N}$, Ardisson Korat AV, de Goede J, et al.; Cohorts for Heart and Aging Research in Genomic Epidemiology (CHARGE) Fatty Acids and Outcomes Research Consortium (FORCE). Omega- 6 fatty acid biomarkers and incident type 2 diabetes: pooled analysis of individuallevel data for 39740 adults from 20 prospective cohort studies. Lancet Diabetes Endocrinol. 2017 Dec;5(12):965-74.

11 Jones PM, Burns CJ, Belin VD, RoderigoMilne HM, Persaud SJ. The role of cytosolic phospholipase $\mathrm{A}(2)$ in insulin secretion. Diabetes. 2004 Feb;53 Suppl 1:S172-8.

12 Persaud SJ, Muller D, Belin VD, Kitsou-Mylona I, Asare-Anane H, Papadimitriou A, et al. The role of arachidonic acid and its metabolites in insulin secretion from human islets of langerhans. Diabetes. 2007 Jan;56(1): 197-203.

13 Kröger J, Zietemann V, Enzenbach C, Weikert C, Jansen EH, Döring F, et al. Erythrocyte membrane phospholipid fatty acids, desaturase activity, and dietary fatty acids in relation to risk of type 2 diabetes in the European Prospective Investigation into Cancer and Nutrition (EPIC)-Potsdam Study. Am J Clin Nutr. 2011 Jan;93(1):127-42.

14 Weber J, Bochi VC, Ribeiro CP, Victório AdM, Emanuelli T. Effect of different cooking methods on the oxidation, proximate and fatty acid composition of silver catfish (Rhamdia quelen) fillets. Food Chem. 2008; 106(1):140-46.

15 Farnetti S, Malandrino N, Luciani D, Gasbarrini G, Capristo E. Food fried in extravirgin olive oil improves postprandial insulin response in obese, insulin-resistant women. J Med Food. 2011 Mar;14(3):316-21.

16 Davies NM, Holmes MV, Davey Smith G. Reading Mendelian randomisation studies: a guide, glossary, and checklist for clinicians. BMJ. 2018 Jul;362:k601.

17 Pingault JB, O’Reilly PF, Schoeler T, Ploubidis GB, Rijsdijk F, Dudbridge F. Using genetic data to strengthen causal inference in observational research. Nat Rev Genet. 2018 Sep;19(9):566-80.

18 Pierce BL, Burgess S. Efficient design for Mendelian randomization studies: subsample and 2-sample instrumental variable estimators. Am J Epidemiol. 2013 Oct;178(7): 1177-84.
19 Hemani G, Zheng J, Elsworth B, Wade KH, Haberland V, Baird D, et al. The MR-Base platform supports systematic causal inference across the human phenome. eLife. 2018 May; $7:$ e34408.

20 Guan W, Steffen BT, Lemaitre RN, Wu JH, Tanaka T, Manichaikul A, et al. Genomewide association study of plasma N6 polyunsaturated fatty acids within the cohorts for heart and aging research in genomic epidemiology consortium. Circ Cardiovasc Genet. 2014 Jun;7(3):321-31.

21 Zerbino DR, Achuthan P, Akanni W, Amode MR, Barrell D, Bhai J, et al. Ensembl 2018. Nucleic Acids Res. 2018 Jan;46 D1:D754-61.

22 Utzschneider KM, Prigeon RL, Faulenbach MV, Tong J, Carr DB, Boyko EJ, et al. Oral disposition index predicts the development of future diabetes above and beyond fasting and 2-h glucose levels. Diabetes Care. 2009 Feb;32(2):335-41.

23 Hartwig FP, Davies NM, Hemani G, Davey Smith G. Two-sample Mendelian randomization: avoiding the downsides of a powerful, widely applicable but potentially fallible technique. Int J Epidemiol. 2016 Dec;45(6): 1717-26.

24 Lemaitre RN, Tanaka T, Tang W, Manichaikul A, Foy M, Kabagambe EK, et al. Genetic loci associated with plasma phospholipid n-3 fatty acids: a meta-analysis of genome-wide association studies from the CHARGE Consortium. PLoS Genet. 2011 Jul;7(7):e1002193.

25 Meyer BJ, Mann NJ, Lewis JL, Milligan GC, Sinclair AJ, Howe PR. Dietary intakes and food sources of omega- 6 and omega- 3 polyunsaturated fatty acids. Lipids. 2003 Apr; 38(4):391-8

26 Zhao JV, Schooling CM. Effect of linoleic acid on ischemic heart disease and its risk factors: a Mendelian randomization study. BMC Med. 2019 Mar;17(1):61.

27 Lawlor DA, Harbord RM, Sterne JA, Timpson N, Davey Smith G. Mendelian randomization: using genes as instruments for making causal inferences in epidemiology. Stat Med. 2008 Apr;27(8):1133-63.

28 Brion MJ, Shakhbazov K, Visscher PM. Calculating statistical power in Mendelian randomization studies. Int J Epidemiol. 2013 Oct;42(5):1497-501.

29 Hewitt J, Walters M, Padmanabhan S, Dawson J. Cohort profile of the UK Biobank: diagnosis and characteristics of cerebrovascular disease. BMJ Open. 2016 Mar; 6(3): e009161.

30 Teumer A. Common methods for performing Mendelian randomization. Front Cardiovasc Med. 2018 May;5:51.

31 Zulyniak MA, Roke K, Gerling C, Logan SL, Spriet LL, Mutch DM. Fish oil regulates blood fatty acid composition and oxylipin levels in healthy humans: A comparison of young and older men. Mol Nutr Food Res. 2016 Mar;60(3):631-41.
32 Li ZH, Zhong WF, Liu S, Kraus VB, Zhang YJ, Gao X, et al. Associations of habitual fish oil supplementation with cardiovascular outcomes and all cause mortality: evidence from a large population based cohort study. BMJ. 2020 Mar;368:m456.

33 Yuan S, Larsson SC. Association of genetic variants related to plasma fatty acids with type 2 diabetes mellitus and glycaemic traits: a Mendelian randomisation study. Diabetologia. 2020;63(1):116-23.

34 Machiela MJ, Chanock SJ. LDlink: a webbased application for exploring populationspecific haplotype structure and linking correlated alleles of possible functional variants. Bioinformatics. 2015 Nov;31(21):3555-7.

35 Willemsen G, Ward KJ, Bell CG, Christensen $\mathrm{K}$, Bowden J, Dalgård $\mathrm{C}$, et al. The Concordance and Heritability of Type 2 Diabetes in 34,166 Twin Pairs From International Twin Registers: The Discordant Twin (DISCOTWIN) Consortium. Twin Res Hum Genet. 2015 Dec;18(6):762-71.

36 Ivarsdottir EV, Steinthorsdottir V, Daneshpour MS, Thorleifsson G, Sulem P, Holm H, et al. Effect of sequence variants on variance in glucose levels predicts type 2 diabetes risk and accounts for heritability. Nat Genet. 2017;49(9):1398-402.

37 Hastert TA, de Oliveira Otto MC, LêScherban F, Steffen BT, Steffen LM, Tsai MY, et al. Association of plasma phospholipid polyunsaturated and trans fatty acids with body mass index: results from the Multi-Ethnic Study of Atherosclerosis. Int J Obes. 2018;42(3):433-40.

38 Flannagan KS, Gahagan S, Das AK, Burrows RA, Lozoff B, Villamor E. Serum Polyunsaturated Fatty Acids in Middle Childhood and Body Mass Index Change Through Adolescence. Ann Nutr Metab. 2019;75(1):77-85.

39 Kelley DS, Taylor PC, Nelson GJ, Mackey BE. Arachidonic acid supplementation enhances synthesis of eicosanoids without suppressing immune functions in young healthy men. Lipids. 1998 Feb;33(2):125-30.

40 Zhou J, Chen L, Liu Z, Sang L, Li Y, Yuan D. Changes in erythrocyte polyunsaturated fatty acids and plasma eicosanoids level in patients with asthma. Lipids Health Dis. 2018 2018/09/01;17(1):206.

41 Cheng L, Zhuang H, Ju H, Yang S, Han J, Tan $\mathrm{R}$, et al. Exposing the causal effect of body mass index on the risk of type 2 diabetes mellitus: a Mendelian randomization study. Front Genet. 2019 Feb;10:94.

42 Wainberg M, Mahajan A, Kundaje A, McCarthy MI, Ingelsson E, Sinnott-Armstrong $\mathrm{N}$, et al. Homogeneity in the association of body mass index with type 2 diabetes across the UK Biobank: A Mendelian randomization study. PLoS Med. 2019 Dec; 16(12):e1002982. 
43 Psaty BM, O’Donnell CJ, Gudnason V, Lunetta KL, Folsom AR, Rotter JI, et al.; CHARGE Consortium. Cohorts for Heart and Aging Research in Genomic Epidemiology (CHARGE) Consortium: design of prospective meta-analyses of genome-wide association studies from 5 cohorts. Circ Cardiovasc Genet. 2009 Feb;2(1):73-80.
44 Scott RA, Lagou V, Welch RP, Wheeler E, Montasser ME, Luan Ja, et al. Large-scale association analyses identify new loci influencing glycemic traits and provide insight into the underlying biological pathways. Nat Genet. 2012;44(9):991-1005.

45 Elliott KS, Chapman K, Day-Williams A, Panoutsopoulou K, Southam L, Lindgren $\mathrm{CM}$, et al.; GIANT consortium; arcOGEN consortium. Evaluation of the genetic overlap between osteoarthritis with body mass index and height using genome-wide association scan data. Ann Rheum Dis. 2013 Jun; 72(6):935-41.
46 Sudlow C, Gallacher J, Allen N, Beral V, Burton P, Danesh J, et al. UK biobank: an open access resource for identifying the causes of a wide range of complex diseases of middle and old age. PLoS Med. 2015 Mar; 12(3):e1001779.

47 Pan A, Teng GG, Yuan J-M, Koh W-P. Bidirectional association between diabetes and gout: the Singapore Chinese Health Study. Sci Rep. 2016;6(1):25766. 